\title{
Melanoma maligno palpebral en un equino: reporte de un caso
}

\section{Malignant melanoma in a equine: report of a case}

\author{
Navarro Cesar A. ${ }^{1}$, Rodríguez Magda E. ${ }^{2}$ y Fuentes Edgar E. ${ }^{2}$ \\ ${ }^{1}$ Medicos Veterinarios y Zootecnistas Unillanos \\ ${ }^{2}$ MVZ. MSc. PhD Docente Unillanos \\ cesar.navarro@unillanos.edu.co
}

Recibido 10 de Diciembre 2011, Aprobado 22 de Febrero 2012

\section{RESUMEN}

El objetivo de este trabajo fue relatar un caso de melanoma en un equino cruceto cuarto de milla, macho, de 10 años de edad, color moro, el cual fue llevado a la clínica de la Universidad de los Llanos por una masa en el parpado derecho que imposibilitaba su visión por dicho ojo, en Marzo del 2010. En este relato de caso, enfatizamos en signos clínicos; exámenes paraclínicos, tales como hemograma, biopsia excisional e histopatología, así como el tratamiento instaurado al animal.

Palabras claves: Melanoma, biopsia excisional, parpado, caballo.

\begin{abstract}
ABSTRAC
The aim of this study is to report a case of melanoma in a quarter-mile horse cross, male, 10 years of age, moor color, which was taken to the clinic of the Universidad de los Llanos by a mass in the right eyelid which prevented his vision for that eye in March 2010. In this case report, we emphasize on clinical signs, laboratory tests such as $\mathrm{CBC}$, excisional biopsy and histopathology, and introduced to animal treatment.
\end{abstract}

Keywords: Melanoma, excisional biopsy, eyelid, horse.

\section{INTRODUCCIÓN}

El melanoma maligno $(\mathrm{MM})$ es un tumor con alta capacidad de generar metástasis a cualquier órgano (Essner et al., 2006). Los melanomas son procesos 
neoplásicos que resultan de alteraciones en los melanócitos o melanoblastos y generalmente se presentan como masas únicas o múltiples localizadas en el tejido dermo-epidermal o subcutáneo. En general, estos tumores se expanden rápidamente o pueden tornarse tumores latentes por largos períodos de tiempo, dependiendo probablemente de la reducida actividad de los melanócitos dérmicos, o a que se presentan encapsulados y disminuye la ocurrencia de metástasis. En otros casos, se diseminan y se tornan letales (Seltenhammer et al., 2003).

Una teoría inicial de la neoplasia melanocítica equina sugiere que los tumores melanocíticos dérmicos y viscerales son manifestaciones de un desorden en el almacenamiento en lugar de una neoplasia maligna y ocurren como resultado del acúmulo de melanina en los melanófagos durante el proceso de despigmentación (Smith et al., 2002). Este disturbio ocurre debido al estímulo de formación de nuevos melanoblastos o aumento de su actividad, resultando en áreas de sobreposición de pigmento en la dermis llevando, consecuentemente, a la falta da transferencia de melanina de los melanócitos de la dermis a las células foliculares, pudiendo estimular variaciones hiperplásicas en las células y su eventual transformación neoplásica (Goetz et al., 1990). Con esto, los tumores más amplios causan obstrucción física del esfínter anal, pene, prepucio o comisura vulvar, y pueden resultar en disquezia, disúria y dificultades en el coito y en el parto. Los tumores melanocíticos pequeños no necesitan ser tratados, en los casos que no estén causando signos clínicos que comprometa la función del respectivo órgano (Rowe y Sullins, 2004).

De modo general, no hay cura para la enfermedad avanzada con metástasis distante. Diversos tipos de tratamiento han sido utilizados en equinos con melanoma, tales como excisión quirúrgica amplia, quimioterapia, radioterapia, además de crionecrosis única o repetida (Rowe y Sullins, 2004; Dummer et al., 1998). La excisión no ha sido recomendada para el tratamiento de melanoma dermal, varios autores han sugerido que la remoción de los tumores melanocíticos, al redor del ano o que involucra la región ventral da la cauda, está 
contra-indicada por causa de imposibilidad de remoción completa, además del estímulo para el crecimiento más rápido del tejido neoplásico o metástasico postquirúrgico (Rowe y Sullins, 2004).

\section{RELATO DEL CASO}

El caso se presenta en un equino macho de 11 años de edad, el cual es un cruceto de cuarto de milla, dicho animal es de color moro, con un peso de $413 \mathrm{~kg}$ (Figura 1), procedente del municipio de Acacias del departamento del Meta, Colombia; el paciente fue llevado a consulta a la Universidad de los Llanos ubicada en la Vereda Barcelona a $12 \mathrm{~km}$ de Villavicencio en el departamento del Meta, por una masa de consistencia dura, indolora, móvil, de aproximadamente 3 $\mathrm{cm}$ de diámetro (Figura 2) en la conjuntiva del parpado superior derecho que impedía la visión del paciente. En la anamnesis el propietario reportó un aumento de tamaño progresivo de la masa desde hace un año. Presentaba epifora en el ojo derecho, además respecto al sistema musculo esquelético, se encontró que el animal era estevado del miembro anterior derecho (MAD) e izquierdo de miembro posterior izquierdo (MPI). El resto del examen físico fue normal, además no se evidenciaron adenopatías regionales.

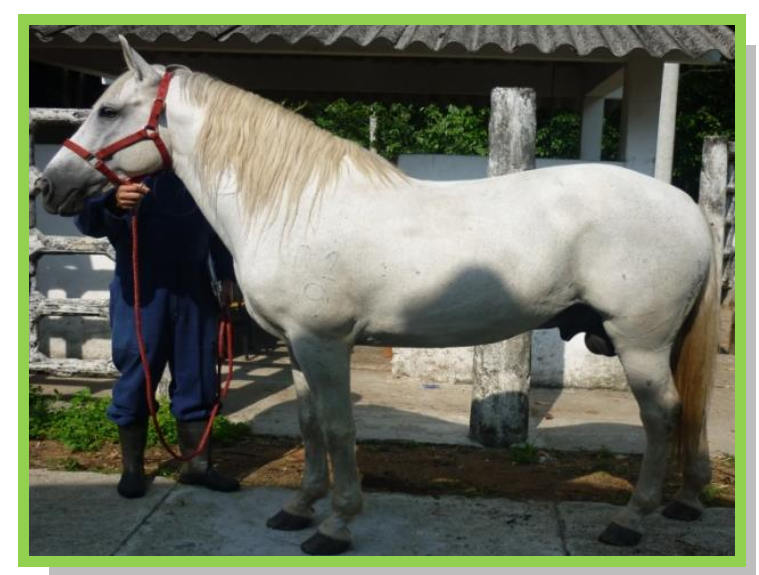

Figura 1. Paciente: Equino macho

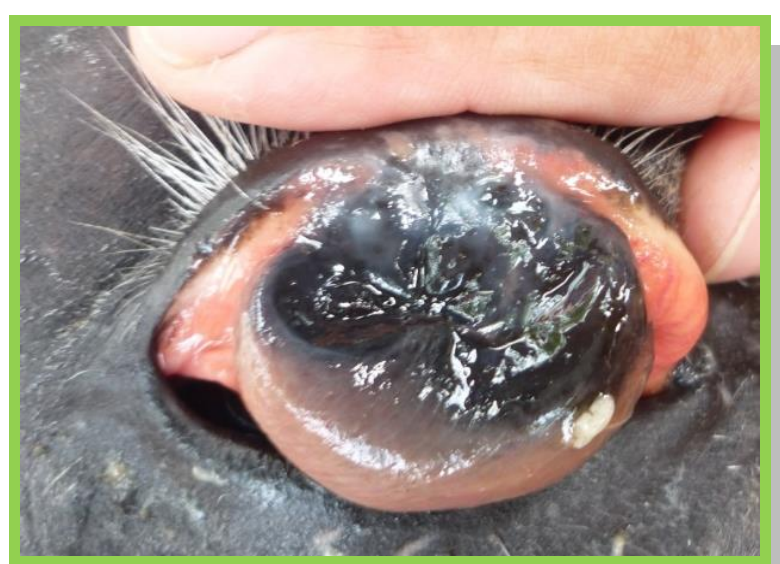

Figura 2. Masa en la mucosa palpebral derecha 
Con la impresión diagnostica de melanoma se realizó una biopsia excisional (Figura 3), obteniendo un resultado anatomo-patológico de melanoma maligno (Figura 4). Los exámenes paraclínicos complementarios como cuadro hemático fueron normales.

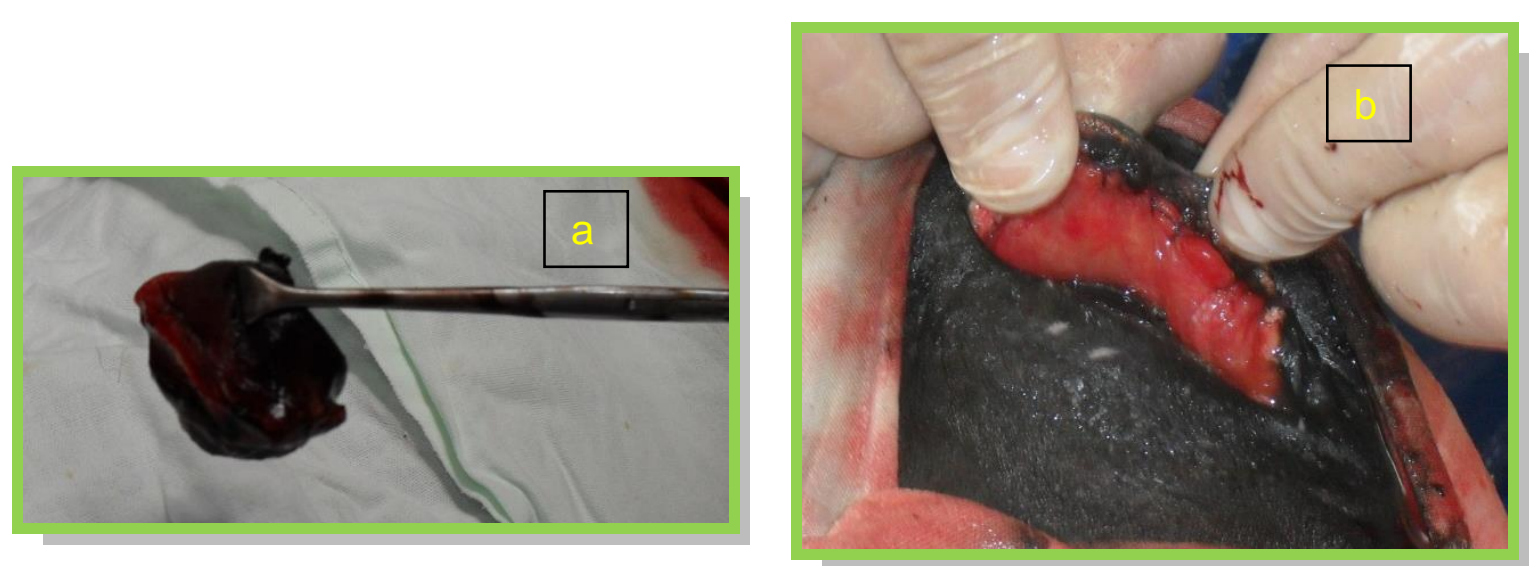

Figura 3. Biopsia excisional. a. Masa excisionada. b. Reconstrucción palpebral
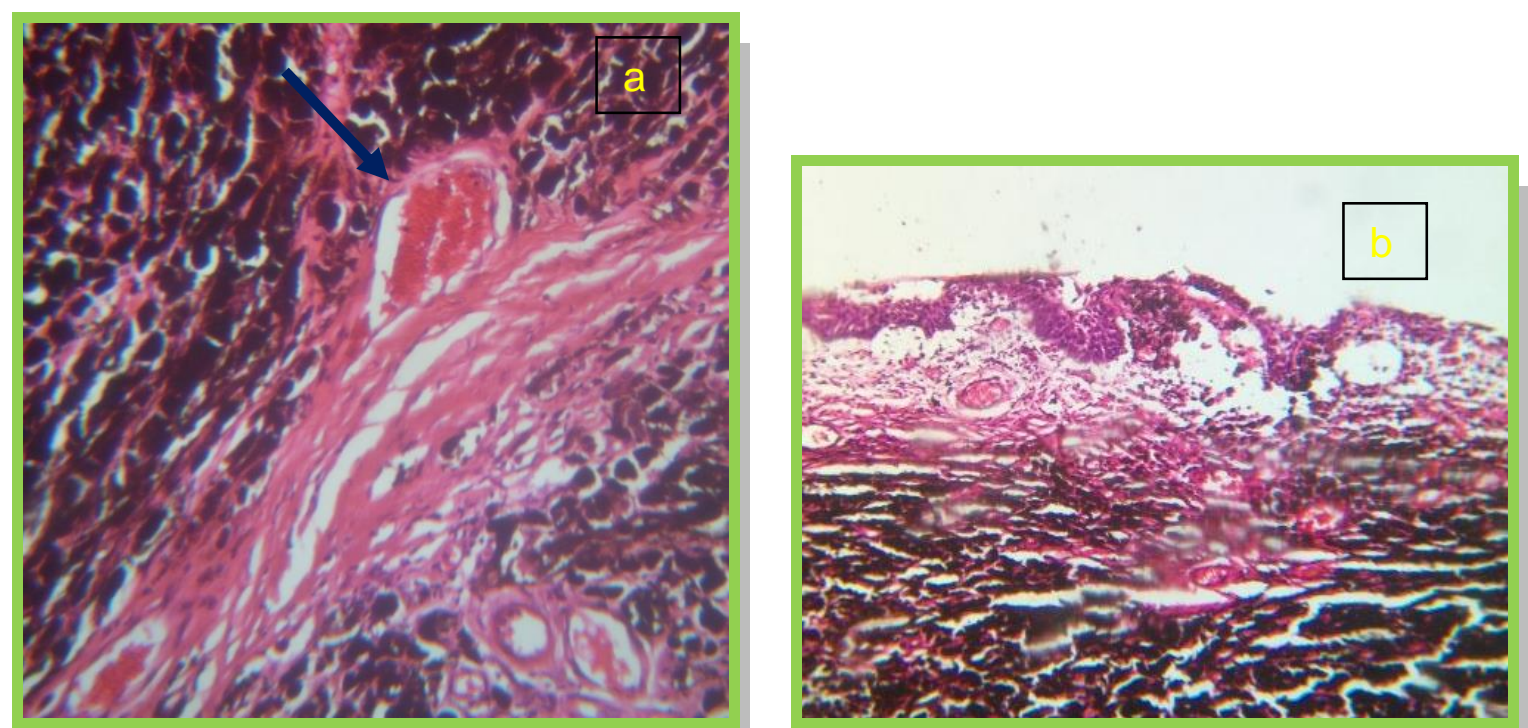

Figura 4. Melanoma maligno. a. La fecha indica la invasión de los melanócitos en el endotelio vascular y muy posiblemente invasión en la luz capilar. b. Invasión epitelial

El tratamiento médico postoperatorio del paciente se realizo con Ceftiofur sódico: 2 mg/kg cada $12 \mathrm{Hrs}$ durante 4 días; Flumixin meglumine: $1,1 \mathrm{mg} / \mathrm{kg}$ cada $12 \mathrm{Hrs}$ durante 3 días; limpieza de la región con Clorhexidina al 0,05\% cada 8 horas; 
Terramicina tópica en la conjuntiva cada $8 \mathrm{Hrs}$ durante 4 días; y Domosyn gel tópico peripalpebral.

Posteriormente el animal fue dado de alta a los 5 días, con una respuesta satisfactoria al tratamiento, pero con un pronóstico reservado ya que el diagnostico histopatológico fue un melanoma altamente maligno.

\section{DISCUSIÓN}

La gran mayoría de melanomas aparecen en los caballos grises o blanco, además las razas árabes, Lipizzanos y percherones parecen tener una predilección por melanoma cutáneo y son poco frecuentes en los caballos de menos de 6 años de edad, los caballos grises desarrollan formas malignas alrededor de los 15 años de edad. (Rowe y Sullins, 2004).

La exposición crónica a la radiación ultravioleta es el factor de riesgo más importante en su iniciación, aunque también presenta predisposición genética. Dentro de los factores promotores se encuentran alteraciones en los melanócitos además de los melanócitomas subcutáneos (Rowe y Sullins, 2004).

Los factores de transformación están dados básicamente por la alteración en los factores supresores de la proliferación celular normal y factores activadores de la apoptosis, por ejemplo, la proteína del retinoblastoma $(\mathrm{Rb})$ y p53, son finalmente superados por los factores de crecimiento no regulados o los receptores del factor de crecimiento (o ambos) y los inhibidores de la apoptosis; por ejemplo, p53 desencadena la detención del ciclo celular o apoptosis previniendo el mantenimiento de las mutaciones del ADN en cualquier línea celular dado. Las mutaciones en los genes INK4a, INK4b, y WAF-1 son más comunes (Seltenhammer et al., 2003; Smith et al., 2002). INK4a y INK4b codifican proteínas p16INK4a y p15INK4b que son inhibidores de la quinasa dependiente de ciclina (CDKI) regulados por $\mathrm{Rb}$. La entrada en la mitosis es desencadenada por ciclinas dependientes de quinasas, por lo tanto, INK4a y mutaciones INK4b en última 
instancia, elimina la capacidad de inhibir la mitosis y permitir la proliferación incontrolada. INK4a también codifica p19ARF, que es un activador de p53. (Rowe y Sullins, 2004; Ritt et al., 1998).

El crecimiento del melanoma esta dado por el factor básico de crecimiento de fibroblastos (bFGF), las interleuquinas (IL): IL-8, IL-10, IL-18, el factor de crecimiento derivado de las plaquetas, y la hormona estimulante de melanócitos (Huang et al., 1999; Lazar-Molnar et al., 2000; Meier et al., 2000; Vidal-Vanaclocha et al., 2000). En las etapas finales de los melanomas son estimulados también por factores tales como la IL- 6 y factor de crecimiento tumoral- $\beta$ que normalmente son inhibidoras de la proliferación de melanócitos (Rowe y Sullins, 2004; Lazar-Molnar et al., 2000).

La metástasis se basa en múltiples procesos que tienen inicio en el desprendimiento de la masa primaria, el movimiento a través del endotelio y, los viajes a través de la sangre o la linfa (o ambos), la adhesión y el movimiento a través del endotelio y, finalmente, el apego y la proliferación dentro de un sitio secundario. Además, se debe tener en cuenta que las células neoplásicas deben regular la expresión de moléculas de adhesión, en el caso de melanoma las dichas moléculas son Cadherina E y CD44 (Hsu et al., 2000; Meier et al., 1998; Sanders et al., 1999). Es muy importante tener en cuenta que durante este proceso complejo de las células tumorales deben escapar a la vigilancia inmunológica, y ser físicamente capaces de sobrevivir y avanzar en el sistema circulatorio o linfático (o ambos), y ser capaces de proliferar en un entorno diferente al estroma de su origen. Una teoría actual sugiere que la evasión del sistema inmune se ve facilitada por la producción de IL-10 en los tumores (Rowe y Sullins, 2004; Huang et al., 1999).

\section{REFERENCIAS BIBLIOGRÁFICAS}

1. Dummer, R.; Hauschild, A.; Henseler, T. et al. Combined interferon - and interleukin - 2 as adjuvant treatment for melanoma. Lancet, London, 352: 908909. 1998. 
2. Essner, R.; Belhocine, T.; Scott, A. M.; Even-Sapir E. Novel imaging techniques in melanoma. Surg Oncol Clin N Am.,15 (2): 253-83. 2006.

3. Goetz, T. E.; Ogilvie, G. K.; Keegan, K. G. et al. Cimetidine for treatment of melanomas in three horses. Journal of the American Veterinary Medical Association, Schaumburg. 196 (3): 449-452. 1990.

4. Hsu, M. Y.; Meier, F. E.; Nesbit, M. et al. E-cadherin expression in melanoma cells restores keratinocyte-mediated growth control and down-regulates expression of invasion-related adhesion receptors. Am Journal Pathology, 156: 1515-1525. 2000.

5. Huang S.; Ullrich, S. E.; Bar-Eli, M. Regulation of tumor growth and metastasis by interleukin-10: the melanoma experience. Journal of Interferon Cytokine, 19: 697-703. 1999.

6. Lazar M., E.; Hegyesi, H.; Toth, S.; Falus, A. Autocrine and paracrine regulation by cytokines and growth factors in melanoma. Journal of Cytokine, 12: 547-554. 2000.

7. Meier, F.; Nesbit, M.; Hsu, M. Y. et al. Human melanoma progression in skin reconstructs: biological significance of bFGF. Am Journal of Pathology, 156: 193-200. 2000.

8. Meier, F.; Satyamoorthy, K. Nesbit, M. et al. Molecular events in melanoma development and progression. Front Biosci, 3: 1005-1010. 1998.

9. Ritt, M. G.; Wojcieszyn, J.; Modiano, J. F. Functional loss of p21/Waf-1 in a case of benign canine multicentric melanoma. Veterinary Pathology, 35: 94101. 1998.

10. Rowe, E. L.; Sullins, K. E. Excision as treatment of dermal melanomatosis in horses: 11 cases (1994 - 2000). Journal of the American Veterinary Medical Association, Schaumburg. 225 (1): 94-96. 2004.

11. Sanders, D. S.; Blessing, K.; Hassan, G. A. et al. Alterations in cadherin and catenin expression during the biological progression of melanocytic tumours. Mol Pathol, 52: 151-157. 1999.

12. Seltenhammer, M. H.; Simhofer, H.; Scherzer, S. et al. Equine melanoma in a population of 296 grey Lipizzaner horses. Equine Veterinary Journal, London. 35 (2): 153-157. 2003.

13. Smith, S. H.; Goldschmidt, M. H.; McManus, P. M. A comparative review of melanocitic neoplasms. Veterinary Pathology, Washington. 39: 651-678. 2002.

14. Vidal-Vanaclocha, F.; Fantuzzi, G.; Mendoza, L. et al. IL-18 regulates IL1 beta-dependent hepatic melanoma metastasis via vascular cell adhesion molecule-1. Proc Natl Acad Sci USA. 97: 734-739. 2000. 